\title{
A saturation theorem for combinations of Bernstein-Durrmeyer polynomials
}

\author{
by P. N. Agrawal and Vijay Gupta $\dagger$ (Roorkee)
}

Abstract. We prove a local saturation theorem in ordinary approximation for combinations of Durrmeyer's integral modification of Bernstein polynomials.

Introduction. The Bernstein-Durrmeyer polynomial of order $n$ is defined by

$$
M_{n}(f, x)=\int_{0}^{1} W(n, x, t) f(t) d t, \quad f \in L_{1}[0,1]
$$

where

$$
W(n, x, t)=(n+1) \sum_{\nu=0}^{n} p_{n \nu}(x) p_{n \nu}(t),
$$

$p_{n \nu}(x)$ being $\left(\begin{array}{l}n \\ \nu\end{array}\right) x^{\nu}(1-x)^{n-\nu}, x \in[0,1]$. These operators were introduced by Durrmeyer [5] by replacing $f(\nu / n)$ in $B_{n}(f, x)$, the Bernstein polynomials, by $(n+1) \int_{0}^{1} p_{n \nu}(t) f(t) d t$. Several authors (see [1]-[4], [6], [8], [9]) have studied the operators $M_{n}$ and obtained direct and inverse results both in supnorm and $L_{p}$-norm. In this paper we study the saturation behaviour of the linear combination $M_{n}(f, k, x)$ [7]. It turns out that even though BernsteinDurrmeyer polynomials are not exponential type operators [7] yet their saturation behaviour is similar to that of the operators of exponential type.

The linear combination $M_{n}(f, k, x)$ of $M_{d_{j} n}(f, x), j=0,1, \ldots, k$, is defined by

$$
M_{n}(f, k, x)=\sum_{j=0}^{k} C(j, k) M_{d_{j} n}(f, x)
$$

1991 Mathematics Subject Classification: 41A30, 41A36.

Key words and phrases: linear combinations, compact support, inner product.

$\dagger$ Research supported in part by Grant No. 5829-11-61 from Council of Scientific and Industrial Research, India. 
where

$$
C(j, k)=\prod_{\substack{i=0 \\ i \neq j}}^{k} \frac{d_{j}}{d_{j}-d_{i}} \quad \text { for } k \neq 0, \quad C(0,0)=1,
$$

and $d_{0}, d_{1}, \ldots, d_{k}$ are $k+1$ arbitrary but fixed distinct positive integers.

R e mark. The definition of $C(j, k)$ can also be related to the formula

$$
x^{k}=\sum_{j=0}^{k} C(j, k) \prod_{\substack{i=0 \\ i \neq j}}^{k}\left(x-d_{i}\right),
$$

which is a simple rephrasing of the interpolation formula

$$
x^{k}=\sum_{j=0}^{k} C(j, k) l_{j}(x),
$$

where $l_{0}, l_{1}, \ldots, l_{k}$ are the Lagrange fundamental polynomials corresponding to the knots $d_{0}, d_{1}, \ldots, d_{k}$. Therefore, by simple computation, we get the relation

$$
\sum_{j=0}^{k} C(j, k)=1 .
$$

Throughout this paper, let $C_{0}$ denote the set of continuous functions on $[0,1]$ having a compact support and $C_{0}^{k}$ the subset of $C_{0}$ of $k$ times continuously differentiable functions. The spaces A.C. $[a, b]$ and $L_{B}[0,1]$ are defined as the class of absolutely continuous functions on $[a, b]$ for every $a, b$ satisfying $0<a<b<1$ and the class of bounded and integrable functions on $[0,1]$ respectively. $\langle a, b\rangle \subset[0,1]$ stands for an open interval containing the closed interval $[a, b]$.

2. Preliminary results. In the following result we obtain an estimate of the degree of approximation by $M_{n}(\cdot, k, x)$ for smooth functions.

ThEOREM 2.1. Let $0 \leq p \leq 2 k+2, f \in L_{B}[0,1]$, and suppose $f^{(p)}$ exists and is continuous on $\langle a, b\rangle \subset[0,1]$. Then for all $n$ sufficiently large,

$$
\left\|M_{n}(f, k, \cdot)-f(\cdot)\right\|_{C[a, b]} \leq \max \left\{C_{1} n^{-p / 2} \omega\left(f^{(p)}, n^{-1 / 2}\right), C_{2} n^{-(k+1)}\right\}
$$

where $C_{1}=C_{1}(k, p), C_{2}=C_{2}(k, p, f)$ and $\omega\left(f^{(p)}, \delta\right)$ denotes the modulus of continuity of $f^{(p)}$ on $\langle a, b\rangle$.

Proof. For $x \in[a, b]$, writing

$$
F(t, x)=f(t)-\sum_{j=0}^{p} \frac{f^{(j)}(x)}{j !}(t-x)^{j},
$$


we have

$$
|F(t, x)| \leq \frac{|t-x|^{p}}{p !}\left(1+\frac{|t-x|}{n^{-1 / 2}}\right) \omega\left(f^{(p)}, n^{-1 / 2}\right)
$$

for $t \in\langle a, b\rangle$. Thus if $\chi(t)$ denotes the characteristic function of $\langle a, b\rangle$, by [3, Prop. II.3] and the Schwarz inequality,

$$
M_{n}(|F(t, x)| \chi(t), x) \leq C_{3} n^{-p / 2} \omega\left(f^{(p)}, n^{-1 / 2}\right),
$$

where $C_{3}=C_{3}(p)$. Similarly, for some constant $C_{4}$ and all $n$ sufficiently large, we have

$$
M_{n}(|F(t, x)|(1-\chi(t)), x) \leq C_{4}\left[M_{n}\left((t-x)^{2(2 k+2)}, x\right)\right]^{1 / 2} \leq C_{5} n^{-(k+1)} .
$$

Hence,

$$
\left|M_{n}(|F(t, x)|, x)\right| \leq C_{3} n^{-p / 2} \omega\left(f^{(p)}, n^{-1 / 2}\right)+C_{5} n^{-(k+1)} .
$$

But by $[6$, Prop. $]$

$$
M_{n}\left(\sum_{j=1}^{p} \frac{f^{(j)}(x)}{j !}(t-x)^{j}, k, x\right)=O\left(n^{-(k+1)}\right)
$$

uniformly in $x \in[a, b]$. Hence for all $n$ sufficiently large

$$
\left\|M_{n}(f, k, \cdot)-f(\cdot)\right\|_{C[a, b]} \leq C_{6} n^{-p / 2} \omega\left(f^{(p)}, n^{-1 / 2}\right)+C_{7} n^{-(k+1)},
$$

where $C_{6}$ does not depend on $f$, from which the required result is immediate.

In the following lemma, the inner product $\langle h(\cdot), g(\cdot)\rangle$ is defined as $\int_{0}^{1} h(x) g(x) d x$.

Lemma 2.2. Let $0<a<b<1$. If $f \in C[0,1]$ and $g \in C_{0}^{\infty}$ with $\operatorname{supp} g \subset(a, b)$, then

$$
\left|n^{k+1}\left\langle M_{2 n}(f, k, \cdot)-M_{n}(f, k, \cdot), g(\cdot)\right\rangle\right| \leq K\|f\|_{C[0,1]},
$$

where $K$ is a constant independent of $f$ and $n$.

Proof. We write

$$
M_{2 n}(f, k, x)-M_{n}(f, k, x)=\sum_{j=1}^{2 k+2} \alpha(j, k) M_{e_{j} n}(f, x),
$$

where $e_{j} \in\left\{d_{0}, d_{1}, \ldots, d_{k}, 2 d_{0}, 2 d_{1}, \ldots, 2 d_{k}\right\}$. By [7, Lemma 3.5] it follows that

$$
\sum_{j=1}^{2 k+2} \alpha(j, k) e_{j}^{-m}=0, \quad m=0,1, \ldots, k .
$$


Next, by using [3, Prop. II.3], we have

$$
\begin{aligned}
n^{k+1}\left\langle M_{2 n}(f, k, \cdot)\right. & \left.-M_{n}(f, k, \cdot), g(\cdot)\right\rangle \\
& =n^{k+1} \int_{0}^{1} \int_{0}^{1}\left\{\sum_{j=1}^{2 k+2} \alpha(j, k) W\left(e_{j} n, x, t\right) f(t) g(x)\right\} d t d x \\
& =n^{k+1} \int_{\operatorname{supp} g} \int_{0}^{1}\{\ldots\} d t d x \\
& =n^{k+1} \int_{\operatorname{supp}} \int_{a}^{b}\{\ldots\} d t d x+o(1)\|f\|_{C[0,1]} \\
& =n^{k+1} \int_{0}^{1} \int_{a}^{b}\{\ldots\} d t d x+o(1)\|f\|_{C[0,1]} .
\end{aligned}
$$

Now, using Fubini's theorem and expanding $g(x)$ by Taylor's theorem, we get

$$
\begin{aligned}
& n^{k+1}\left\langle M_{2 n}(f, k, \cdot)-M_{n}(f, k, \cdot), g(\cdot)\right\rangle \\
& =n^{k+1} \int_{a}^{b} \int_{0}^{1} \sum_{i=0}^{2 k+2} \sum_{j=1}^{2 k+2} \frac{\alpha(j, k)}{i !} W\left(e_{j} n, x, t\right) f(t) g^{(i)}(t)(x-t)^{i} d x d t \\
& \quad+n^{k+1} \int_{a}^{b} \int_{0}^{1} \sum_{j=1}^{2 k+2} \alpha(j, k) W\left(e_{j} n, x, t\right) f(t) \varepsilon(x, t)(x-t)^{2 k+2} d x d t \\
& \quad+o(1)\|f\|_{C[0,1]} \\
& =\sum_{i=0}^{2 k+2} n^{k+1} \int_{a}^{b} \int_{0}^{1} \sum_{j=1}^{2 k+2} \frac{\alpha(j, k)}{i !} W\left(e_{j} n, x, t\right) f(t) g^{(i)}(t)(x-t)^{i} d x d t \\
& \quad+n^{k+1} \int_{0}^{1} \int_{a}^{b} \sum_{j=1}^{2 k+2} \alpha(j, k) W\left(e_{j} n, x, t\right) f(t) \varepsilon(x, t)(x-t)^{2 k+2} d t d x \\
& \quad+o(1)\|f\|_{C[0,1]} \\
& =J_{1}+J_{2}+o(1)\|f\|_{C[0,1]}, \quad \text { say },
\end{aligned}
$$

where $\varepsilon(x, t)(x-t)^{2 k+2}$ is the remainder term corresponding to the partial Taylor expansion of $g$.

Since, for $\xi$ lying between $t$ and $x$,

$$
|\varepsilon(x, t)|=\frac{\left|g^{(2 k+2)}(\xi)-g^{(2 k+2)}(x)\right|}{(2 k+2) !} \leq \frac{2}{(2 k+2) !}\left\|g^{(2 k+2)}\right\|_{C[a, b]}<\infty,
$$


using [3, Prop. II.3], it follows that $J_{2}=O(1)\|f\|_{C[0,1]}$.

To estimate $J_{1}$, we proceed as follows: $J_{1}$ may be rewritten as

$$
J_{1}=n^{k+1} \sum_{i=0}^{2 k+2} \frac{1}{i !} \sum_{j=1}^{2 k+2} \alpha(j, k) \int_{a}^{b}\left(\int_{0}^{1} W\left(e_{j} n, x, t\right)(x-t)^{i} d x\right) f(t) g^{(i)}(t) d t
$$

Now, we note that $W(n, x, t)=W(n, t, x)$, therefore using [3, Prop. II.3], after interchanging the variables $t$ and $x$, together with equation (2.1) it follows that $J_{1}=O(1)\|f\|_{C[0,1]}$. Combining the estimates for $J_{1}, J_{2}$ and (2.2), we obtain the required result.

Theorem 2.3 [2]. Let $f \in C[0,1], 0<a_{1}<a_{2}<a_{3}<b_{3}<b_{2}<b_{1}<1$ and $0<\alpha<2$. Then, in the following, the implications (i) $\Rightarrow($ ii) $\Leftrightarrow($ iii) $\Rightarrow$ (iv) hold:

(i) $\left\|M_{n}(f, k, \cdot)-f(\cdot)\right\|_{C\left[a_{1}, b_{1}\right]}=O\left(n^{-\alpha(k+1) / 2}\right)$.

(ii) $f \in \operatorname{Liz}\left(\alpha, k+1, a_{2}, b_{2}\right)$.

(iii) (a) For $m<\alpha(k+1)<m+1, m=0,1 \ldots, 2 k+1, f^{(m)}$ exists and is is in $\operatorname{Lip}\left(\alpha(k+1)-m, a_{2}, b_{2}\right)$.

(b) For $\alpha(k+1)=m+1, m=0,1, \ldots, 2 k, f^{(m)}$ exists and is in $\operatorname{Lip}^{*}\left(1, a_{2}, b_{2}\right)$.

(iv) $\left\|M_{n}(f, k, \cdot)-f(\cdot)\right\|_{C\left[a_{3}, b_{3}\right]}=O\left(n^{-\alpha(k+1) / 2}\right)$.

Here $\operatorname{Liz}(\alpha, k, a, b)$ denotes the class of functions for which $\omega_{2 k}(f, h, a, b)$ $\leq M h^{\alpha k}$; when $k=1, \operatorname{Liz}(\alpha, 1)$ reduces to the Zygmund class Lip* $\alpha$.

\section{The saturation result}

TheOREM 3.1. Let $f \in C[0,1]$ and $0<a_{1}<a_{2}<a_{3}<b_{3}<b_{2}<b_{1}<1$. Then, in the following statements, the implications (i) $\Rightarrow($ ii $) \Rightarrow$ (iii) and (iv) $\Rightarrow$ (v) $\Rightarrow$ (vi) hold true:

(i) $n^{k+1}\left\|M_{n}(f, k, \cdot)-f(\cdot)\right\|_{C\left[a_{1}, b_{1}\right]}=O(1)$;

(ii) $f^{(2 k+1)} \in A . C .\left[a_{2}, b_{2}\right] \quad$ and $f^{(2 k+2)} \in L_{\infty}\left[a_{2}, b_{2}\right]$;

(iii) $n^{k+1}\left\|M_{n}(f, k, \cdot)-f(\cdot)\right\|_{C\left[a_{3}, b_{3}\right]}=O(1)$;

(iv) $n^{k+1}\left\|M_{n}(f, k, \cdot)-f(\cdot)\right\|_{C\left[a_{1}, b_{1}\right]}=o(1)$;

(v) $f \in C^{2 k+2}\left[a_{2}, b_{2}\right] \quad$ and $\sum_{j=1}^{2 k+2} \frac{Q(j, k, x)}{j !} f^{(j)}(x)=0, \quad x \in\left[a_{2}, b_{2}\right]$,

where $Q(j, k, x)$ are the polynomials occurring in [6, Th. 2];

(vi) $n^{k+1}\left\|M_{n}(f, k, \cdot)-f(\cdot)\right\|_{C\left[a_{3}, b_{3}\right]}=o(1)$,

where all $O(1)$ and $o(1)$ terms are with respect to $n$, as $n \rightarrow \infty$. 
Proof. First assume (i); then in view of (i) $\Rightarrow$ (iii) of Theorem 2.3 , it follows that $f^{(2 k+1)}$ exists and is continuous on $\left(a_{1}, b_{1}\right)$. Moreover, the statement

$$
\left\|M_{n}(f, k, \cdot)-f(\cdot)\right\|_{C\left[a_{1}, b_{1}\right]}=O\left(n^{-(k+1)}\right)
$$

is equivalent to

$$
\left\|M_{2 n}(f, k, \cdot)-M_{n}(f, k, \cdot)\right\|_{C\left[a_{1}, b_{1}\right]}=O\left(n^{-(k+1)}\right) .
$$

Indeed, trivially $(3.1) \Rightarrow(3.2)$. Also, assuming $(3.2)$, since $\lim _{n \rightarrow \infty} M_{n}(f, k, x)$ $=f(x)$, we can write

$$
\begin{aligned}
f(x)=M_{n}(f, k, x) & +\left[M_{2 n}(f, k, x)-M_{n}(f, k, x)\right] \\
& +\left[M_{4 n}(f, k, x)-M_{2 n}(f, k, x)\right]+\ldots \\
& +\left[M_{2^{r} n}(f, k, x)-M_{2^{r-1} n}(f, k, x)\right]+\ldots
\end{aligned}
$$

Hence,

$$
\begin{aligned}
&\left\|f(\cdot)-M_{n}(f, k, \cdot)\right\|_{C\left[a_{1}, b_{1}\right]} \\
& \leq\left\|M_{2 n}(f, k, \cdot)-M_{n}(f, k, \cdot)\right\|_{C\left[a_{1}, b_{1}\right]} \\
& \quad+\left\|M_{4 n}(f, k, \cdot)-M_{2 n}(f, k, \cdot)\right\|_{C\left[a_{1}, b_{1}\right]} \\
& \quad+\ldots+\left\|M_{2^{r} n}(f, k, \cdot)-M_{2^{r-1} n}(f, k, \cdot)\right\|_{C\left[a_{1}, b_{1}\right]}+\ldots \\
&= K_{1}\left(\frac{1}{n^{k+1}}+\frac{1}{2^{k+1} n^{k+1}}+\ldots+\frac{1}{\left(2^{r-1}\right)^{k+1} n^{k+1}}+\ldots\right) \\
&= \frac{K_{1}}{n^{k+1}} \frac{1}{1-2^{-(k+1)}}=\frac{K_{2}}{n^{k+1}},
\end{aligned}
$$

where $K_{2}=K_{1} /\left(1-2^{-(k+1)}\right)$, showing that (3.1) holds.

Thus, we may assume that $\left\{n^{k+1}\left(M_{2 n}(f, k, \cdot)-M_{n}(f, k, \cdot)\right)\right\}$ is bounded as a sequence in $C\left[a_{1}, b_{1}\right]$ and hence in $L_{\infty}\left[a_{1}, b_{1}\right]$. Since $L_{\infty}\left[a_{1}, b_{1}\right]$ is the dual space of $L_{1}\left[a_{1}, b_{1}\right]$, it follows by Alaoglu's theorem that there exists $h \in L_{\infty}\left[a_{1}, b_{1}\right]$ such that for some subsequence $\left\{n_{i}\right\}_{i=1}^{\infty}$ of natural numbers and for every $g \in C_{0}^{\infty}$ with $\operatorname{supp} g \subset\left(a_{1}, b_{1}\right)$

$$
\lim _{n_{i} \rightarrow \infty} n_{i}^{k+1}\left\langle M_{2 n_{i}}(f, k, \cdot)-M_{n_{i}}(f, k, \cdot), g(\cdot)\right\rangle=\langle h(\cdot), g(\cdot)\rangle .
$$

Now, since $C^{2 k+2}\left[a_{1}, b_{1}\right] \cap C[0,1]$ is dense in $C[0,1]$ there exists a sequence $\left\{f_{\sigma}\right\}_{\sigma=1}^{\infty}$ in $C^{2 k+2}\left[a_{1}, b_{1}\right] \cap C[0,1]$ converging to $f$ in $\|\cdot\|_{C[0,1]}$-norm. Then, for any $g \in C_{0}^{\infty}$ with $\operatorname{supp} g \subset\left(a_{1}, b_{1}\right)$ and each function $f_{\sigma}$, by [6, Th. 2] we have 


$$
\begin{aligned}
\lim _{n_{i} \rightarrow \infty} n_{i}^{k+1}\left\langle M_{2 n_{i}}\left(f_{\sigma}, k, \cdot\right)-M_{n_{i}}\left(f_{\sigma}, k, \cdot\right), g(\cdot)\right\rangle \\
=\left\langle-\left(1-2^{-(k+1)}\right) \sum_{j=1}^{2 k+2} \frac{Q(j, k, \cdot)}{j !} f_{\sigma}^{(j)}(\cdot), g(\cdot)\right\rangle \\
=\left\langle P_{2 k+2}(D) f_{\sigma}(\cdot), g(\cdot)\right\rangle=\left\langle f_{\sigma}(\cdot), P_{2 k+2}^{*}(D) g(\cdot)\right\rangle,
\end{aligned}
$$

where $P_{2 k+2}^{*}(D)$ denotes the operator adjoint to $P_{2 k+2}(D)$ (in this case, it is simply a result of integration by parts). By Lemma 2.2, we conclude that

$$
\begin{array}{r}
\lim _{n_{i} \rightarrow \infty} n_{i}^{k+1}\left|\left\langle M_{2 n_{i}}\left(f-f_{\sigma}, k, \cdot\right)-M_{n_{i}}\left(f-f_{\sigma}, k, \cdot\right), g(\cdot)\right\rangle\right| \\
\leq K\left\|f-f_{\sigma}\right\|_{C[0,1]} .
\end{array}
$$

Hence, by (3.5), (3.4) and (3.3) (in that order)

$$
\begin{aligned}
& \left\langle f(\cdot), P_{2 k+2}^{*}(D) g(\cdot)\right\rangle=\lim _{\sigma \rightarrow \infty}\left\langle f_{\sigma}(\cdot), P_{2 k+2}^{*}(D) g(\cdot)\right\rangle \\
& =\lim _{\sigma \rightarrow \infty}\left\{\lim _{n_{i} \rightarrow \infty} n_{i}^{k+1}\left\langle M_{2 n_{i}}\left(f-f_{\sigma}, k, \cdot\right)-M_{n_{i}}\left(f-f_{\sigma}, k, \cdot\right), g(\cdot)\right\rangle\right. \\
& \left.+\left\langle f_{\sigma}(\cdot), P_{2 k+2}^{*}(D) g(\cdot)\right\rangle\right\} \\
& =\lim _{n_{i} \rightarrow \infty} n_{i}^{k+1}\left\langle M_{2 n_{i}}(f, k, \cdot)-M_{n_{i}}(f, k, \cdot), g(\cdot)\right\rangle=\langle h(\cdot), g(\cdot)\rangle,
\end{aligned}
$$

for all $g \in C_{0}^{\infty}$ with $\operatorname{supp} g \subset\left(a_{1}, b_{1}\right)$. Thus

$$
P_{2 k+2}(D) f(x)=h(x)
$$

as generalized functions.

Note that $Q(2 k+2, k, x) \neq 0$ by [6, Prop.]. Therefore, regarding (3.6) as a first order linear differential equation for $f^{(2 k+1)}$, we deduce that $f^{(2 k+1)} \in$ A.C. $\left[a_{2}, b_{2}\right]$ and hence $f^{(2 k+2)} \in L_{\infty}\left[a_{2}, b_{2}\right]$. This completes the proof of the implication (i) $\Rightarrow$ (ii).

Now assuming (ii), it follows that $f^{(2 k+1)} \in \operatorname{Lip}_{M}\left(1, a_{2}, b_{2}\right)$ with $M=$ $\left\|f^{(2 k+2)}\right\|_{L_{\infty}\left[a_{2}, b_{2}\right]}$. Hence (iii) follows by Theorem 2.1 .

To prove (iv) $\Rightarrow(\mathrm{v})$, assuming (iv) and proceeding in the manner of the proof of (i) $\Rightarrow$ (ii), we get $P_{2 k+2}(D) f(x)=0$, from which in view of the non-vanishing of $Q(2 k+2, k, x)$, (v) is clear.

The proof of $(\mathrm{v}) \Rightarrow$ (vi) follows from [6, Th. 2]. This completes the proof of the theorem.

Acknowledgement. The authors are extremely grateful to the referee for the critical review of the paper. 


\section{References}

[1] P. N. Agrawal and V. Gupta, Simultaneous approximation by linear combination of the modified Bernstein polynomials, Bull. Soc. Math. Grèce 30 (1989), 21-29 (1990).

[2] - , - Inverse theorem for linear combinations of modified Bernstein polynomials, preprint.

[3] M. M. Derriennic, Sur l'approximation de fonctions intégrables sur $[0,1]$ par des polynômes de Bernstein modifiés, J. Approx. Theory 31 (1981), 325-343.

[4] Z. Ditzian and K. Ivanov, Bernstein-type operators and their derivatives, ibid. 56 (1989), 72-90.

[5] J. L. Durrmeyer, Une formule d'inversion de la transformée de Laplace: Application à la théorie des moments, Thèse de 3e cycle, Faculté des Sciences de l'Université de Paris, 1967.

[6] H. S. Kasana and P. N. Agrawal, On sharp estimates and linear combinations of modified Bernstein polynomials, Bull. Soc. Math. Belg. Sér. B 40 (1) (1988), 61-71.

[7] C. P. May, Saturation and inverse theorems for combinations of a class of exponential type operators, Canad. J. Math. 28 (1976), 1224-1250.

[8] B. Wood, $L_{p}$-approximation by linear combinations of integral Bernstein-type operators, Anal. Numér. Théor. Approx. 13 (1) (1984), 65-72.

[9] - Uniform approximation by linear combinations of Bernstein-type polynomials, J. Approx. Theory 41 (1984), 51-55.

DEPARTMENT OF MATHEMATICS

UNIVERSITY OF ROORKEE

ROORKEE 247667, U.P., INDIA
Current address of P. N. Agrawal: DEPARTMENT OF MATHEMATICS MOI UNIVERSITY ELDORET, KENYA 